\title{
Experimental measurements of sediment incipient velocity by using B-scan ultrasound imaging device in the water channel
}

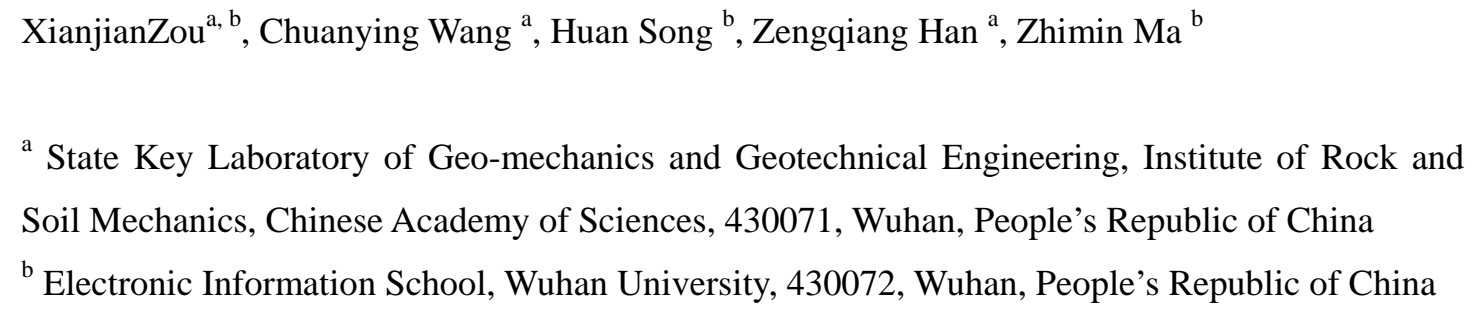

Abstract: Sediment incipient velocity (SIV) is an important parameter for the rule of sediment movement and fluvial processes in the river sediment engineering. The field measurement of the SIV during the process of sediment incipient motion near the riverbed is a difficult problem. For this problem, this paper puts forward a novel method of measuring SIV by using B-scan ultrasound imaging device and current meter in the water channel of river modelexperiment. In this method, we use a B-scan ultrasound imaging device to get the ultrasonic images of moving particles and riverbed under the water, and use a current meter to obtain the flow velocities of observed points near the bed at the same time. By statistical analysis of the images, relationships between the imaging spot number/areaof particles and the water flow velocityare obtained, which have shown the whole process of sediment incipient motion clearly. Results show there are some suddenly changed positions in these relationships. These suddenly changed positions correspond to the states of incipient motion, which can be used to analyse and measure the SIV. After kinds of model experiments, the measured SIV is within an acceptable range and can be verified by the change of riverbed boundary lines. This method is very suitable for rivermodel experiments, especially for water channel test under muddy water or sediment-laden flow. This paper provides a new approachtothe research and analysis of sediment movement with the advantages of direct observation and real-time research.

Key words:Incipient motion; river model experiment; sediment incipient velocity; ultrasound imaging measurement; water channel 


\section{Introduction}

Sediment incipient motion is one of fundamental problems in the research of sediment transport theory and also is always first encountered parameter in the study of engineering-related sediments [1-3]. Many scientists had devoted their efforts to the study on the sediment incipient motion and had accumulated abundant datain the past years[4-6].One vital parameter of the data is the sediment incipient velocity (SIV) during the sediment incipient motion. The SIV can be defined as a critical water flow velocity when the sediment particle is going on to move away from the riverbed. And there is little focus on the directand effective methodsfor themeasurement of the SIVin the river model experiment[7, 8]. However, the measurement of the SIV is very difficult and has drawn a great practical significance in the river sediment engineeringduring the model experiment[9]. It is also one of the basic tasks in the river dynamics and sedimentation research[10]. The analysis of the SIV is more difficult especially in the movable-bed model experiment and urgent to adopt anew effective method.

On the research of sediment incipient motion, many incipient velocity formulas were put forward for the estimation of the SIV[11]. Most of them are based on the theoretical analysis of sediment mechanical model, but they are not practical obtained and hardly to be used for local certain problem in practice[12]. Up to now, the direct measurementand analysis of the SIV according to local certain conditions are seldom reported. The SIVis very difficult to be measured accurately with a single uniform formula in actual application becauseit is influenced by material properties, particle sizes and shapes of sediments, and their inner relationships are hard to be determined [13-15]. Actually, SIV is usually measured by manual observation and the analysis of observed flow velocity data according toriverbed conditions under the water during the process of sediment incipient motion[16-19]. This measurement method depends on subjectivity of human greatly and lacks of quantitative criterion. The availability of manual work is limited and hard to spread other situations.Therefore, when water is too muddy to be seen clearly, this traditional method cannot be adopted, and it limits the further research of related issues, such as sediment transport and channel erosion[20].Since high frequency ultrasonic waves can penetrate sediment-laden water flow, which is transmitted from B-scan ultrasound imaging device, we can use 
this B-scan ultrasound imaging device to monitor the incipient motion of sediment under muddy water or turbulent flow[21-24]. In this paper, a novelmethod of measuring the SIV by using B-scan ultrasound imaging technique is proposed and verified through water channel model experiments at Wuhan University.

\section{Experimental system}

High frequency ultrasonic wave is sensitive to underwater sediments or tiny particles, whichis for industrial applications with strong penetrability. Suspended sediments can be monitored very well, especially with the help of modern B-scan ultrasound imaging technology, such as a B-scan ultrasound imaging device SIUI APOGEE 1100 Digital Color Doppler Ultrasound Imaging System.This device can be used to monitor the sediment incipient motion on the river model experiment.It is detailed described as follow.

\subsection{B-scan ultrasound imaging device}

B-scan ultrasound imaging device is widely used in medicine and spread in the river model experimentof sediment engineering.The detector of B-scan ultrasound imaging device generates ultrasonic waves and transmits to water flow. And then the echoes of sediment particles or bottom riverbed are received by the detector. The propagation time of ultrasonic waves is measured electronically and the distance from sediment particles to detector is determined inside. After a series of signal processing steps within B-scan ultrasound imaging device, a profile image of sediment-laden water flow including thesediment particles and the river-bed is formed. The profile imageis called B-scan ultrasonic image.The B-scan ultrasound imaging device which is used in this paper is shown in Fig. 1a.

The detector of used B-scan ultrasound imaging deviceis a convex array probe, which can transmit ultrasonic waves. The frequency of ultrasonic waves can be 2.0 $\mathrm{MHz}, 2.5 \mathrm{MHz}, 3.3 \mathrm{MHz}, 4.2 \mathrm{MHz}$ and $5.0 \mathrm{MHz}$ with the control of inner electronic equipment. The measuring depth of this ultrasound device is less than $40 \mathrm{~cm}$ and the measuring width of device is less than $38 \mathrm{~cm}$. It is because that B-scan ultrasound imaging device is original designed for medical diagnosis. The measuring angle of this detector is from $10^{\circ}$ to $70^{\circ}$. The acquisition frame frequency of this device ranges from $8 \mathrm{~Hz}$ to $63 \mathrm{~Hz}[22]$. 
The ultrasonic waves from B-scan ultrasound imaging device are sensitive to tiny particles under the water. If ultrasonic frequency is suitable for the imaging of sediment particles, the moving particles near the riverbed can be real-time monitored very well[25]. When we use B-scan ultrasound imaging device to monitor the sediment-laden water flow during theriver model experiment, the images of sediment particles and river-bed can be obtained.One of obtained B-scan ultrasonic images is shown in Fig. 1b. This image is aprofile map of sediment-laden flow including the imaging of moving particle and bottom riverbed.

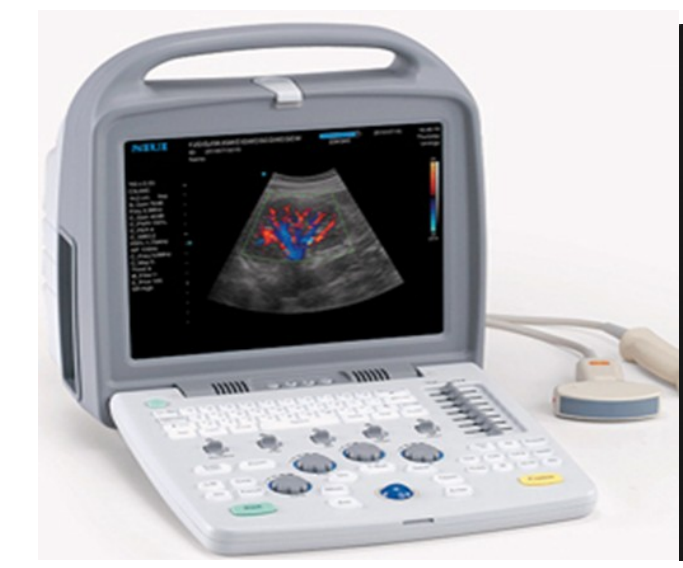

(a)

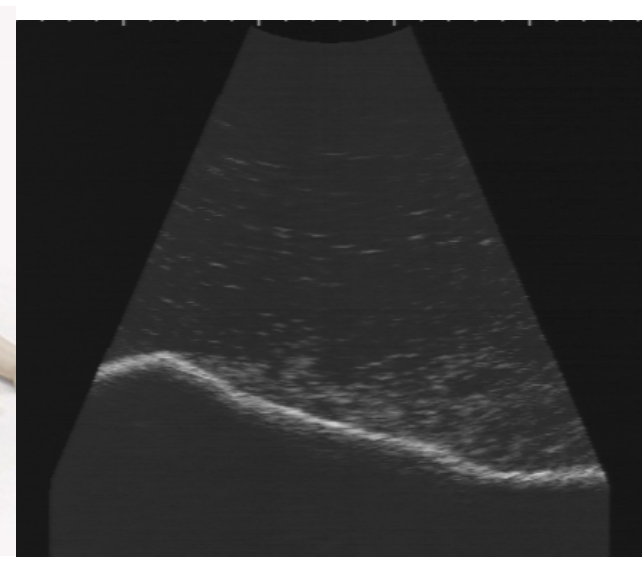

(b)

Figs. 1.B-scan ultrasound imaging device (a) and a typical ultrasonic image(b)for the imaging of moving particles and bottom riverbed in the sediment-laden flow

From Fig. 1b,it is can be seen that the imaging of moving particles and bottom riverbed under the water is monitored clearly. The B-scan ultrasonic image can show the state of sediment motion. The imaging spots of moving particles are observed very well with good self-adaption and high resolution.B-scan ultrasound imaging device is sensitive to moving sediment particles in the water. We can use this device to monitor the underwater sediment motion in real time.

\subsection{System composition}

In order to study the incipient motion of sediment and acquire the ultrasonic images of moving sediment particles near the riverbed, an experimental system is built. This system is consisted of the B-scan ultrasound imaging device, a computer, an acquisition card, a circulating water channel, a current meter and a pump shown in Fig. 2. The size of water channel is $5.0 \mathrm{~m} \times 1.2 \mathrm{~m} \times 1.0 \mathrm{~m}$ (Length $\times$ Width $\times$ Depth) 
The frequency of ultrasonic waves transmitted from the detector of B-scan ultrasound imaging device is $5 \mathrm{MHz}$, which is usually taken by us.

In the system Fig. 2, the pump is used to controlwater flow velocity,and the circulating water channel is used to form a controlled circulating system. The current meter measures and records the real-time flow velocity at the observed point (shown in Fig. 2) every 30 minutes. The bottom of the circulating water channel is covered with model sands (e.g., natural sands and plastic sands).The detector of B-scan ultrasound imaging device is used to obtain the ultrasonic images of sediment-laden flow in the water channel. The acquisition card is used to transmit the images to the computer, which is for the later imageanalysis and measurement.

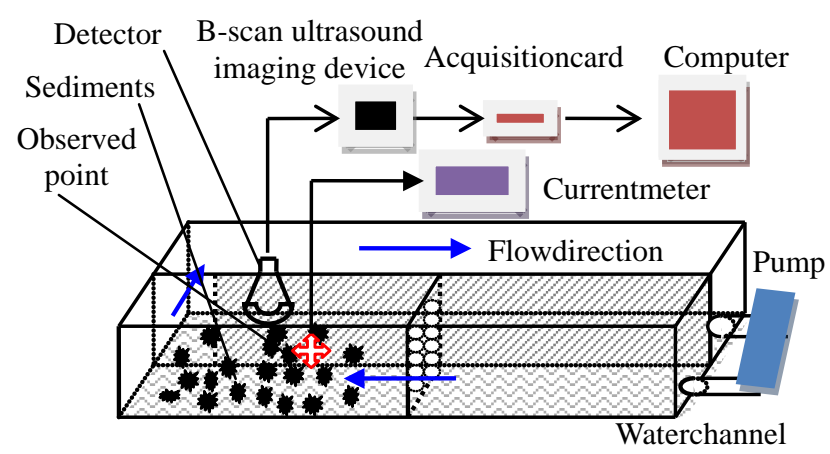

Fig. 2.The experimental system (schematic diagram)

\section{Sediment experiments}

In this section, the application of B-scan ultrasound imaging device by using above experimental system is described in detail. The system's ultrasonic frequency is set as $5 \mathrm{MHz}$ and kept as a constant. When the ultrasonic images are clear, the setting of this system should be kept on and so asother related settings, such as the position of current meter and the TGC settings of B-scan ultrasound imaging device.All of these should remain the same as normal condition until the whole group experiments are finished. Another, model sands refer to plastic sands or natural sands, etc. They are used to formthe moving particles and riverbed during the following experiment.

\subsection{Different sediment particleexperiments}

To illustrate the temporal and spatial resolution of B-scan ultrasound imaging device, different particle sizes of plastic sands and natural sands are used in the model experiment. Some typical ultrasonic images of experimental results are shown in 
154

155

156

157

158

159

160

161

162

Figs.3. The ultrasonic image of plastic sandswhose particle size is $0.1 \mathrm{~mm}$ isshown in Fig. 3a.The ultrasonic image of plastic sands whose particle size is $0.2 \mathrm{~mm}$ isshown in Fig. 3b.The ultrasonic image of natural sands whose particle size is $0.1 \mathrm{~mm}$ isshown in Fig. 3c.The ultrasonic image of natural sands whose particle size is $0.3 \mathrm{~mm}$ isshown in Fig. 3d.Figs. 3have clearlyshownthe suspension or slow movement states ofsediment particlesunder the water.

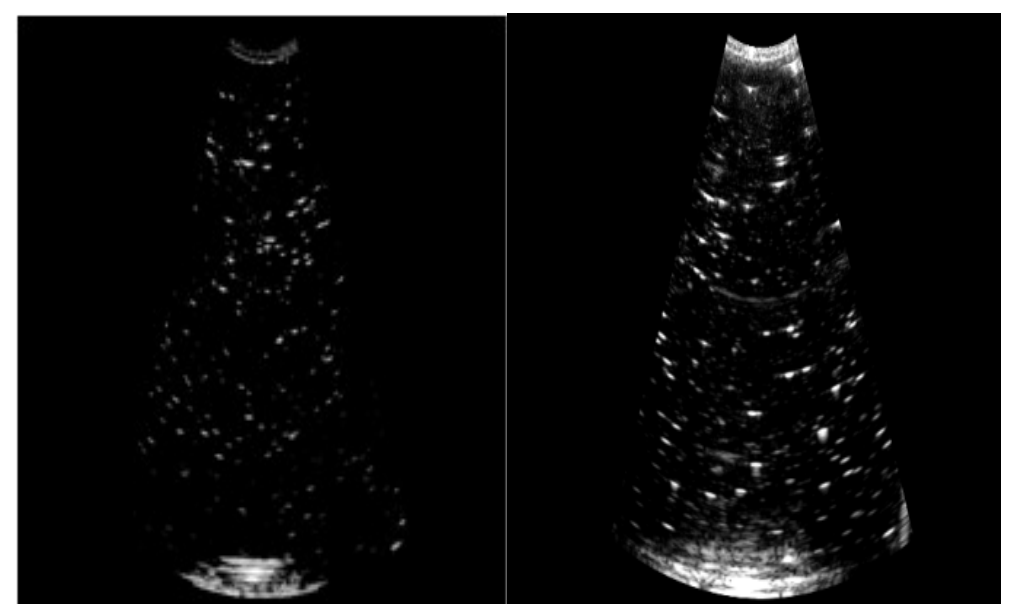

(a)

(b)

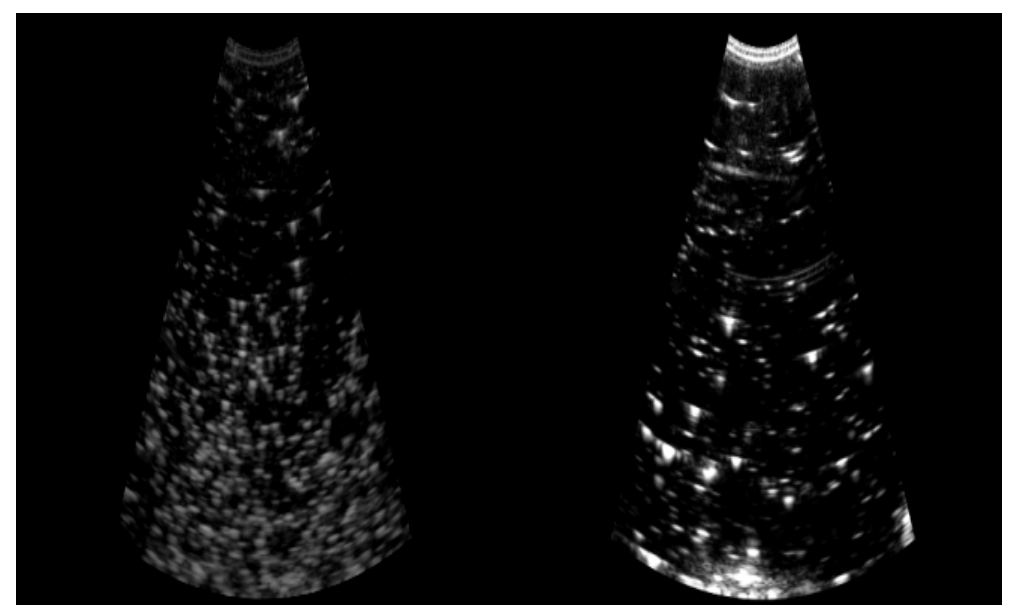

(c)

(d)

Figs. 3.Ultrasonic images from different sediment particles experiments (profile map), (a) is sediment particles mean size $0.1 \mathrm{~mm}$ from plastic sands, (b)is sediment particles mean size $0.2 \mathrm{~mm}$ from plastic sands, (c)is sediment particles mean size $0.1 \mathrm{~mm}$ from natural sands, (d) is sediment particles mean size $0.3 \mathrm{~mm}$ from natural sands.

From Figs. 3, we can see that the imaging spots are larger when the sediment particle sizes are bigger,e.g., Figs. 3a-3d, and the images are both clear for different sediment particles even though particle size is as small as about $0.1 \mathrm{~mm}$. It means that the B-scan ultrasound imaging device has a high temporal and spatial resolution, 
which is high enough to monitor most of suspending particles. Another, the imaging signals of different kinds of material sands at the same particle size are still have some differences, but the main imaging feature is nearly same. For example, the sizes and the number of imaging spots whose material is plastic sandsin Fig. 3a are nearly the same as the sizes and the number of imaging spots whose material is natural sands in Fig. 3c. It means B-scan ultrasound imaging device is sensitive to the particle size of sediments and the effect on the imaging of different kinds of material sediments can be negligible. So, the temporal and spatial resolution of B-scan ultrasound imaging device is enough for the research of sediment incipient motion. This device can be used to monitor the sediment-laden flow.

\subsection{Sediment incipient motionexperiments}

To researchsediment incipient motion and measure the SIV, the experimental system (i.e, Fig. 2) is used to acquire the ultrasonic images of moving sediment particles near the riverbed. We take kinds of model sands for the sedimentincipient motion experiments, such as model plastic sand and model plastic sand. Plastic sands (particle size $0.1 \mathrm{~mm}$ and $0.2 \mathrm{~mm}$ ) and natural sands (particle size $0.1 \mathrm{~mm}$ and 0.3 $\mathrm{mm}$ ) are used in these experiments, respectively. They are taken as examples to show and illustrate the process of sediment incipient motion. Water flow velocity is controlled by the pump and maintained within a certain velocity segment for half an hour. The certain velocity segment is increased every hour from $2.01 \mathrm{~cm} / \mathrm{s}$ to 12.76 $\mathrm{cm} / \mathrm{s}$. These velocity segments are the controlled water flow velocity in the water channel by the pump. The real-time values of flow velocity near the observed point are measured everyminute by the current meter during everysegment. The real-time flow velocity refers to the velocity at the observed point near the riverbed and detector of B-scan ultrasound imaging device, shown in Fig. 2. The mean flow velocity of every segment is obtained during the experiments.

During the experiments, the motion of sediment particles in the water flow becomes more and more obvious when the flow velocity is added by pump every segment[25]. In order to well understand the process of sediment incipient motion, we analyse the number and area of imaging spots from sediment particles persegment by using mathematical statistical methods[26]. The mean flow velocity of every segment during the experiment is denoted by $V$. The mean number of imaging spots from sediment particles in the water flow is denoted by $N$. The mean area of imaging spots 
from sediment particles in the water flow is denoted by $S$. Some related statistical data are shown in table 1 . The mean number of imaging spots from plastic sands whose particle size is $0.1 \mathrm{~mm}$ is short as $N$-ps- 0.1 in the table. The mean area of imaging spots from natural sands whose particle size is $0.3 \mathrm{~mm}$ is short as - -ns-0.3.The meanings of $N$-ns-0.1, $N$-ps-0.2, $N$-ns-0.3, $S$-ps-0.1, $S$-ns-0.1 and $S$-ps-0.2 are similar to $N$-ps-0.1 and $S$-ns-0.3 in the table.

Table 1 Statistical data ofimaging spotsfrom sediment particles under different flow velocity segments

\begin{tabular}{cccccccccc}
\hline$i$ & $V$ & $N$-ps-0.1 & $N$-ns-0.1 & $N$-ps-0.2 & $N$-ns-0.3 & $S$-ps-0.1 & $S$-ns-0.1 & $S$-ps-0.2 & $S$-ns-0.3 \\
\hline 1 & 2.32 & 1.51 & 1.41 & 1.03 & 0.65 & 4.32 & 1.45 & 3.01 & 1.12 \\
2 & 2.86 & 2.22 & 1.64 & 1.31 & 0.81 & 7.06 & 2.53 & 5.27 & 1.82 \\
3 & 3.32 & 3.71 & 2.05 & 1.65 & 1.03 & 11.21 & 4.31 & 7.43 & 2.41 \\
4 & $\mathbf{3 . 9 5}$ & $\mathbf{9 . 6 4}$ & 3.06 & 2.12 & 1.32 & $\mathbf{3 0 . 1 5}$ & 7.45 & 10.35 & 3.45 \\
5 & $\mathbf{4 . 5 1}$ & 14.61 & $\mathbf{7 . 6 2}$ & 2.87 & 1.65 & 45.45 & $\mathbf{1 8 . 7 4}$ & 15.45 & 4.35 \\
6 & $\mathbf{5 . 1 9}$ & 20.55 & 9.95 & $\mathbf{9 . 1 5}$ & 2.45 & 63.01 & 24.85 & $\mathbf{3 7 . 0 1}$ & 9.51 \\
7 & $\mathbf{5 . 7 5}$ & 25.46 & 11.76 & $\mathbf{1 3 . 7 6}$ & $\mathbf{6 . 3 4}$ & 76.67 & 29.14 & $\mathbf{5 3 . 1 6}$ & $\mathbf{2 8 . 9 5}$ \\
8 & 6.29 & 29.98 & 13.41 & 18.08 & 7.98 & 89.43 & 33.26 & 67.64 & 46.81 \\
9 & 6.72 & 33.52 & 14.71 & 21.56 & 9.15 & 100.15 & 37.21 & 79.12 & 60.71 \\
10 & 7.29 & 37.95 & 16.02 & 26.23 & 10.61 & 114.75 & 41.87 & 94.58 & 79.95 \\
11 & 7.81 & 41.95 & 16.95 & 30.56 & 13.55 & 128.31 & 46.25 & 108.97 & 97.25 \\
12 & 8.22 & 44.55 & 17.59 & 34.23 & 14.65 & 141.42 & 49.21 & 117.65 & 112.41 \\
\hline
\end{tabular}

(Note: $V$ refers to the mean flow velocity of every segment; $N$ refers to the mean number of imaging spots from sediment particles in the water flow; $S$ refers to the mean area of imaging spots; ps refers to plastic sands; ns refers to natural sands; $0.1,0.2$ and 0.3 refer to the mean particle sizes of sediment particles; $i$ is the serial number ofexperimental segments)

According to the characteristics of sediment motion process and the definition of the SIV[11], when flow velocity is lower than the SIV, the particles on the riverbed are motionless except for some tiny particles because of water flow conditions. The riverbed and the number of imaging spots from moving sediment particles remain unchanged at this time. When flow velocity reaches or exceeds the SIV of the sediment particles near the bed, the particles will move along river bed or leave the bed. Because the particles are out of mechanical equilibrium, the river bed begins to change. Therefore, the number of imaging particles above theriverbed will increase rapidly. When water flow velocity becomes higher, most of particles start moving. Obviously, the number of moving particles will greatly increase, and the riverbed will 
be changed after a certain period if flow velocity is bigger than the SIV. Therefore, the number of imaging particle spots above the river bed is an important parameter to measure the SIV. And the statistical analysis of imaging area from particles spots can help the further understanding of sediment incipient motion. So, the related statistical analysis of imaging spots number and area are necessary. They are the data basis of sediment incipient motion and can help to the measurement of the SIV. Lastly,the change of riverbed can be used to validate themeasured SIV.

To show the incipient process of sediment motion, we took plastic sands whose average particle size is $0.2 \mathrm{~mm}$ as example to go on a series of experiments. Three instantaneous states during the sediment incipientprocess are shown in Figs. 4. The motionless state of sediment particles is shown in Fig. 4a when water flow velocity is small. If flow velocity is higher, sediment particles start to go on incipient motion near the topographic bed, which is shown in Fig. 4b. Fig. 4c shows the condition ofmany sediment particlesare moving away from the bottom riverbed if water flow velocity is too high.

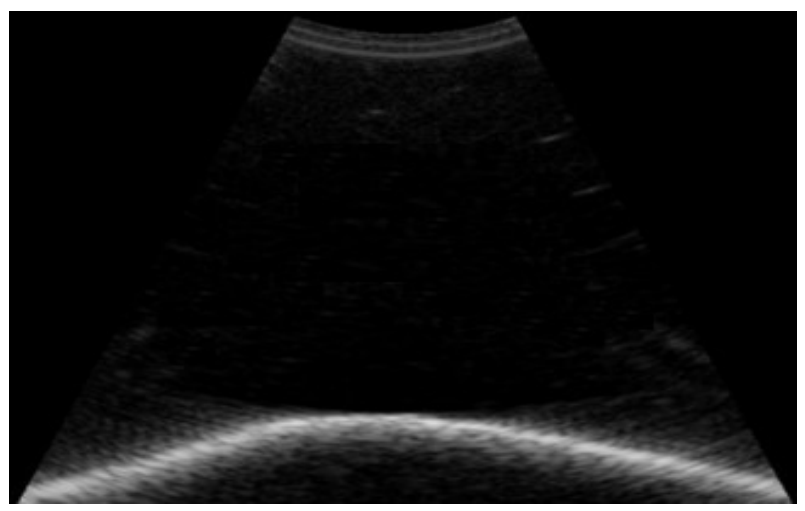

(a)

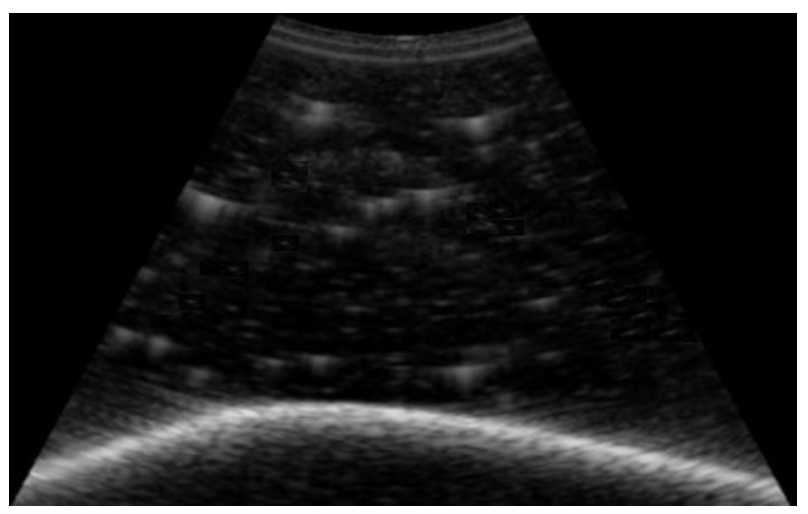

(b) 


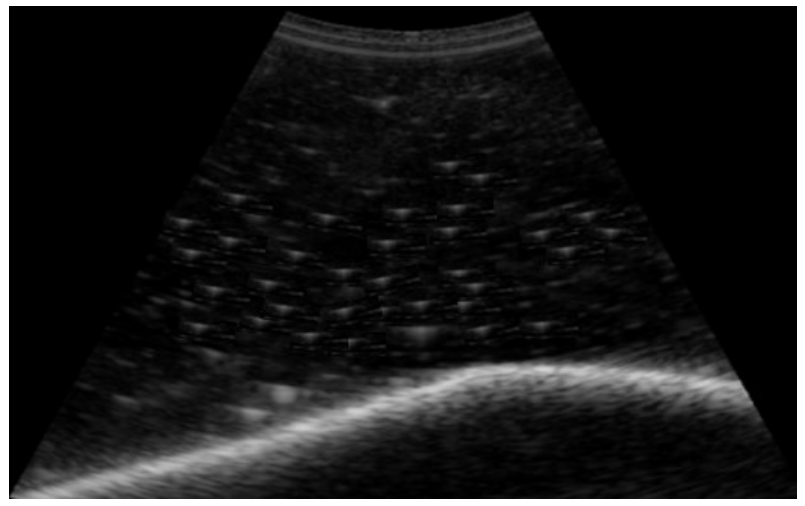

(c)

Figs. 4.Conditions of particles near the riverbed under different flow velocities, (a)sediment particles stand motionless when flow velocity is $2.5 \mathrm{~cm} / \mathrm{s}$, (b)sediment particles start to move when flow velocity is up to $5.8 \mathrm{~cm} / \mathrm{s}$, (c)sediment particles are moving when flow velocity is up to $13.6 \mathrm{~cm} / \mathrm{s}$

From Figs. 4, Fig. 4a is the motionless condition of sediment. Since the flow velocity (e.g., $2.5 \mathrm{~cm} / \mathrm{s}$ ) is too low to let plastic sands move, the particles on the bed cannot move along the riverbed. The number of imaging spots Nis zero and also the area of imaging spots is zero. While the flow velocity is up to $5.8 \mathrm{~cm} / \mathrm{s}$, the sands on the riverbed start to move. At this time, the number of imaging spots increases rapidly, e.g., Fig. 4b, the mean number of imaging spots is 13.76 (mean particles)and the mean area of imaging spots is 53.16 (mean pixel units). So, we can be sure that the sands on the riverbed are going on incipient motion. About 30 minutes later, when the flow velocity is up to $13.6 \mathrm{~cm} / \mathrm{s}$, the riverbed is obviously changed, e.g., Fig. 4c, and the number and area of imaging spots are hugely added.The incipient motion of sediment particlesis very fierce at this time during this flow velocity segment.

Therefore, the change feature of imaging spots (number and area) obtained by B-scan ultrasound imaging device can be used to analyze the incipient motion, and then the obtained corresponding flow velocity of incipient motion is determined as the measured SIV after the analysis of statistical data.

\section{Analysis and discussion}

When flow velocity is added from $2.32 \mathrm{~cm} / \mathrm{s}$ to $5.75 \mathrm{~cm} / \mathrm{s}$, we can be sure that the sediment particles have gone on incipient motion at some points below 5.75 $\mathrm{cm} /$ saccording to Figs. 4.But when and where the sediment particles are going on incipient motion, they should be answered. In order to further understanding the 
feature of incipient motion, the number and area of imaging spots from sediment particles in B-scan ultrasonic images are analyzed and discussed in this section.

\subsection{Analysis of imaging spot number}

To obtain the number of imaging spots in the ultrasonic image in the process of sediment incipient motion, the images are analysed by some image pre-treatment and segmentation methods. Table 1 shows related data of $N$-ps-0.1, $N$-ns-0.1, $N$-ps-0.2 and $N$-ns- 0.3 at different flow velocity segments. The data of $N$-ps- $0.1, N$-ns- $0.1, N$-ps- 0.2 and $N$-ns-0.3 in the table are computed as follow:

$$
N_{i}=\sum_{j=1}^{m} n_{j} / m
$$

wherei is the number of row in Table $1, j$ is the statistical number of the imaging spot number, $n_{j}$ is the imaging spot number value in the ultrasound image when the current meter obtained the flow velocity eachtime, $m$ is the times of every segment. So, $N_{i}$ is the meanvalue of imaging spots' number within $m$ (e.g., $m=100$ ) images during each flow velocity segment.

The computisticalnumber $N_{i}$ according to formula (1) is shown directly in table 1 (i.e., $N$-ps-0.1, $N$-ns- $0.1, N$-ps- 0.2 and $N$-ns-0.3). Their relation curves of $N_{i}$ to flow velocity $V_{i}$ are shown in Fig. 5a.

In order to understand the change rate of imaging spots' number $N_{i}$ and velocity $V$, thechange rateof number is defined as:

$$
R \mathrm{n}_{i}=\frac{\left|N_{i+1}-N_{i}\right| / N_{1}}{\left|V_{i+1}-V_{i}\right| / V_{1}}
$$

where $R \mathrm{n}_{i}$ is the ratio of $N_{i}$ to velocity $V_{i}, i$ is the serial number of segments. So, $R \mathrm{n}_{i}$ is the change rate of imaging spots number every flow velocity segment.

Therefore, the defined change rate of number $R \mathrm{n}_{i}$ can give a deep understanding of sediment incipient motion. The change rate curve of plastic sands' imaging spots number Nis defined as Rn-ps-0.1, which particle size is $0.1 \mathrm{~mm}$ and corresponding to the changed rate of $N$-ps-0.1.The change rate curve ofnatural sands' imaging spots number Nis defined as $R n-n s-0.3$, which particle size is 0.3 mmand corresponding to the changed rate of $N$-ns-0.3. The meaning of $R \mathrm{n}$-ps-0.2 whose particle size is 0.2 mmis the same as $R n-p s-0.1$. Andthe meaning of $R n-n s-0.1$ whose particle size is 0.1 mmis the same as $R \mathrm{n}$-ns-0.3.Their relation curves of $R n_{i}$ to flow velocity $V_{i}$ are shown in Fig. 5b. 


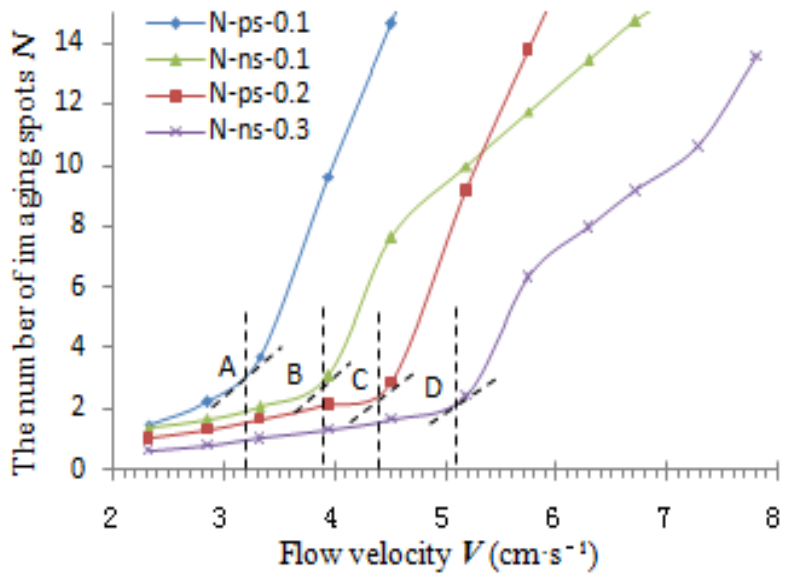

(a)

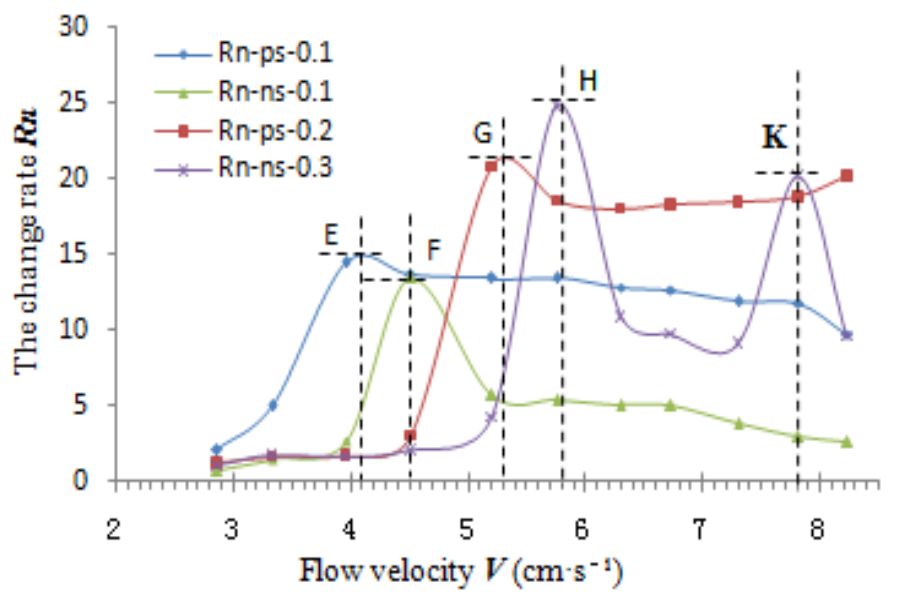

(b)

Figs. 5.The curves of imaging spots number $N$ and their corresponding change rates $R n$,(a)the curves $N$-ps-0.1, $N$-ns- $0.1, N$-ps-0.2and $N$-ns-0.3 ofimaging spots number $N$, (b)the curves $R$ n-ps-0.1, $R n-n s-0.1, R n-p s-0.2$ and $R n$-ns- 0.3 oftheir corresponding change rates $R n$

From table 1 and Fig. 5a, the $N$-ps-0.1, $N$-ns- $0.1, N$-ps- 0.2 and $N$-ns- 0.3 both increase with the increase of the flow velocity $V$. The number of imaging spots $N$-ps-0.1 (i.e., plastic sands) is bigger than $N$-ns- 0.1 (i.e., natural sands) at the same flow velocity and same particle size. This is because the density of plastic sands is smaller than that of natural sands. The $N$-ps-0.2 (plastic sands) is bigger than $N$-ps-0.1 (natural sands) at the same flow velocity and same material. This is because the particle size of $N$-ps- 0.2 is bigger than the particle size of $N$-ps-0.1. Plastic particles with smaller sizesare lighter and easier to move away from river bed. Their curves of $N$-ps-0.1, $N$-ns- $0.1, N$-ps- 0.2 and $N$-ns- 0.3 both have a process of sudden increase, e.g., the tangent points $\mathrm{A}, \mathrm{B}, \mathrm{C}$ and $\mathrm{D}$ shown as Fig. 5a.Their corresponding flow velocities 
of tangent points A, B, C and D are about $3.2 \mathrm{~cm} / \mathrm{s}, 3.8 \mathrm{~cm} / \mathrm{s}, 4.4 \mathrm{~cm} / \mathrm{s}$ and 5.1 $\mathrm{cm} / \mathrm{s}$.And we find after these points, the number of imaging spots increase faster. It means the sediment particles start to go on incipient motion at the velocities of these points. So the velocities of sediment incipient motion (i.e., SIVs) are bigger than the velocities of $3.2 \mathrm{~cm} / \mathrm{s}, 3.8 \mathrm{~cm} / \mathrm{s}, 4.4 \mathrm{~cm} / \mathrm{s}$ and $5.1 \mathrm{~cm} / \mathrm{s}$, respectively.

From Fig. 5b, the change rates $R \mathrm{n}-\mathrm{ps}-0.1, R \mathrm{n}-\mathrm{ns}-0.1, R \mathrm{n}-\mathrm{ps}-0.2$ and $R \mathrm{n}-\mathrm{ns}-0.3$ both have a process of sudden change revulsion calculated according to formula (2). The maximum value points of $R \mathrm{n}-\mathrm{ps}-0.1, R \mathrm{n}-\mathrm{ns}-0.1, R \mathrm{n}-\mathrm{ps}-0.2$ and $R \mathrm{n}-\mathrm{ns}-0.3$ are points E, F, G and $\mathrm{H}$. At these points, the number of imaging spots increases quickly. It means that there are more and more moving particles near the riverbed in the water. Where arethose more and more moving particles from? They are from the sediment particles on the riverbed. What does it mean? It means the sediment particles have gone on incipient motion before these points. So the velocities of sediment incipient motion (i.e., SIVs) are smaller than the velocities of these points. The corresponding flow velocities of points E, F, G and $H$ are about $4.1 \mathrm{~cm} / \mathrm{s}, 4.5 \mathrm{~cm} / \mathrm{s}, 5.3 \mathrm{~cm} / \mathrm{s}$ and 5.8 $\mathrm{cm} / \mathrm{s}$. These velocities have already reached the sediment incipient velocity, i.e., SIV. So, the SIV of $0.1 \mathrm{~mm}$ plastic sands is bigger than $3.2 \mathrm{~cm} / \mathrm{s}$ and smaller than 4.1 $\mathrm{cm} / \mathrm{s}$; the SIV of $0.1 \mathrm{~mm}$ natural sands is bigger than $3.8 \mathrm{~cm} / \mathrm{s}$ and smaller than 4.5 $\mathrm{cm} / \mathrm{s}$; the SIV of $0.2 \mathrm{~mm}$ plastic sands is bigger than $4.4 \mathrm{~cm} / \mathrm{s}$ and smaller than 5.3 $\mathrm{cm} / \mathrm{s}$; and the SIV of $0.3 \mathrm{~mm}$ natural sands is bigger than $5.1 \mathrm{~cm} / \mathrm{s}$ and smaller than 5.8 $\mathrm{cm} / \mathrm{s}$. We can take their mid-value as their SIVs. Therefore, the SIV of $0.1 \mathrm{~mm}$ plastic sands is about $3.7 \mathrm{~cm} / \mathrm{s}(3.2 / 2+4.1 / 2)$; the SIV of $0.1 \mathrm{~mm}$ natural sands is about 4.2 $\mathrm{cm} / \mathrm{s}(3.8 / 2+4.5 / 2)$; the SIV of $0.2 \mathrm{~mm}$ plastic sands is about $4.9 \mathrm{~cm} / \mathrm{s}(4.4 / 2+5.3 / 2)$; and the SIV of $0.3 \mathrm{~mm}$ natural sands is about $5.5 \mathrm{~cm} / \mathrm{s}(5.1 / 2+5.8 / 2)$.

\subsection{Analysis of imaging spot area}

Because of water flow movement and some unsteady scattering of ultrasonic waves, the imaging particles may be unclear to be counted sometimes, especially when the imaging spots are filled with the ultrasound image. To further illustrate the imagingfeaturesof sediment incipient motion, the relationship between the statistical area of imaging spots and the water flow velocity can be calculated according to the following formula:

$$
S_{i}=\sum_{j=1}^{m} S_{j} / m
$$


363

364

365

366

367

368

369

370

371

372

373

374

375

376

377

378

379

380

381

382 wheres $s_{j}$ is the area value of the current meter every minute during the experiment, $m$ is the times of every segment. So, $S_{i}$ is the mean value of imaging spots'areawithin $m$ (e.g., $m=100$ ) images during each flow velocity segment.

The computistical area $S_{i}$ according to formula (3) is shown directly in table 1 (i.e., $S$-ps-0.1,S-ns-0.1,S-ps-0.2 and $S$-ns-0.3). Their relation curves of $S_{i}$ to flow velocity $V_{i}$ are shown inFig. 6a. The change rate of imaging spots' area is also defined as:

$$
R s_{i}=\frac{\left|S_{i+1}-S_{i}\right| / S_{1}}{\left|V_{i+1}-V_{i}\right| / V_{1}}
$$

where $R \mathrm{~s}_{i}$ is the ratio of $N_{i}$ to velocity $V_{i}, i$ is the serial number of segments.

So, $R s_{i}$ is the change rate of imaging spots'area every flow velocity segment.The change rate curve of plastic sands' imaging spots areaSis defined as Rs-ps-0.1, which particle size is $0.1 \mathrm{~mm}$ and corresponding to the changed rate of $S$-ps-0.1.The change rate curve ofnatural sands' imaging spots area $S$ is defined as Rs-ns-0.3, which particle size is 0.3 mmand corresponding to the changed rate of $S$-ns-0.3. The meaning of $R s-p s-0.2$ whose particle size is $0.2 \mathrm{mmis}$ the same as Rs-ps-0.1. Andthe meaning of Rs-ns-0.1whose particle size is $0.1 \mathrm{mmis}$ the same as $R s-n s-0.3$.Their relation curves of $R \mathrm{~s}_{i}$ to flow velocity $V_{i}$ are shown in Fig. $6 \mathrm{~b}$.

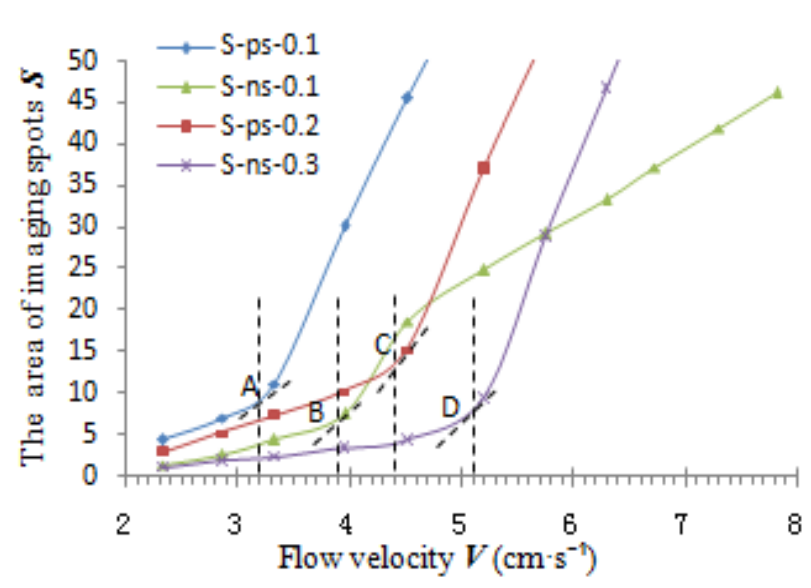

(a) 


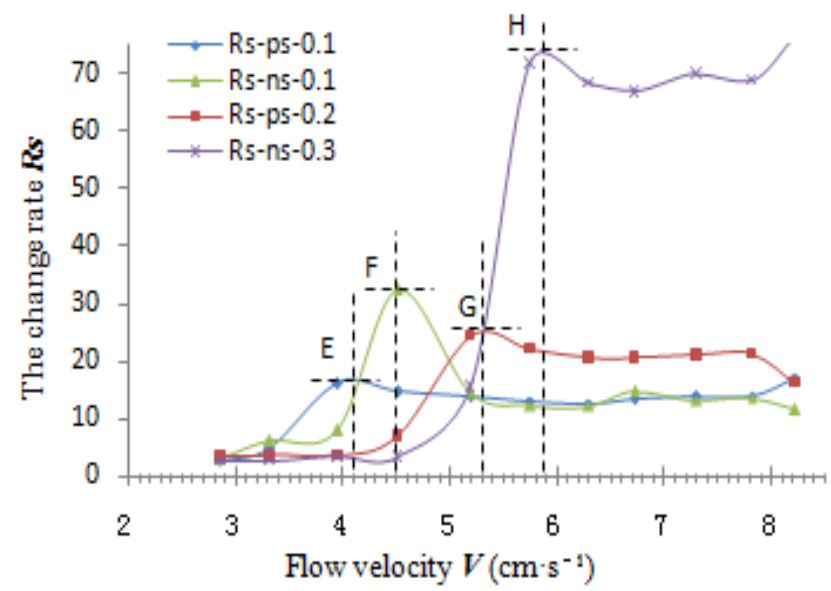

(b)

Figs. 6.The curves of imaging spots area $S$ and their corresponding change rates $R \mathrm{~s}$, (a)the curves S-ps-0.1, S-ns-0.1, S-ps-0.2 and S-ns-0.3 of imaging spots area $S$, (b)the curves Rs-ps-0.1, Rs-ns-0.1, Rs-ps-0.2 and Rs-ns-0.3 oftheir corresponding change rates $R \mathrm{~s}$

From Figs. 6, we can see that the change trends of $S$-ps-0.1, $S$-ns-0.1, $S$-ps-0.2, S-ns-0.3, Rs-ps-0.1, Rs-ns-0.1, Rs-ps-0.2 and Rs-ns-0.3 are similar as the change trends of $N$-ps-0.1, $N$-ns-0.1, $N$-ps-0.2, $N$-ns-0.3, Rn-ps-0.1, Rn-ns-0.1, Rn-ps-0.2 and $R \mathrm{n}-\mathrm{ns}-0.3$. The analysis of Figs. 6 is the same as that of Figs. 5.Therefore, we can get the corresponding velocities $(3.2 \mathrm{~cm} / \mathrm{s}, 3.9 \mathrm{~cm} / \mathrm{s}, 4.4 \mathrm{~cm} / \mathrm{s}$ and $5.1 \mathrm{~cm} / \mathrm{s})$ of tangent points A, B, C and D in Fig. 6a, and also the corresponding velocities $(4.1 \mathrm{~cm} / \mathrm{s}, 4.5$ $\mathrm{cm} / \mathrm{s}, 5.3 \mathrm{~cm} / \mathrm{s}$ and $5.8 \mathrm{~cm} / \mathrm{s}$ ) of points E, F, G and Hin Fig. 6b. These velocities are nearly the same as the obtained velocitiesin Figs. 5. So the corresponding SIVs of Figs. 6are the same as the obtained SIVs of Figs. 5. The measured SIVs of different statistical methods (statistical number and area) are same. It further illustrates the measured SIVs e.g., 3.7 cm/s, 4.2 cm/s, 4.9 cm/s, 5.5 cm/s) of Figs. 5 are correct and reliable.

\subsection{Discussion and uncertainty analysis}

From above, the mean number and area of imaging spots in ultrasound images can effectively reflect the whole process of sediment incipient motion, and the flow velocities of their suddenly increased pointscorrespond to the SIVs, e.g., points $\mathrm{A} \sim \mathrm{H}$ in Figs. 5-6.Therefore, the number and area of imaging spots in the ultrasound images can be used to measure SIV in the river model experiments. However, there are still some difficulties during actual experiments, such as river bed and water flow conditions. Different flow or riverbed conditions can lead to different incipient motion 
states and also result in different SIVs. When river bed is horizontal and smooth, the sediment particles on the riverbed are easiest to go on incipient motion because of high Reynolds number according to Shields diagram and Yang Velocity approaches [27-31]. Instead, when river bed is not horizontal and smooth, the particles on the riverbed are hard to go on incipient motion. Reynolds number and Shields diagram are discussed little here because they had been clearly described in other related references. We show two typical states of sediment movement under different river beds and flowconditions during the model experimentsshown asFigs. 7-8.

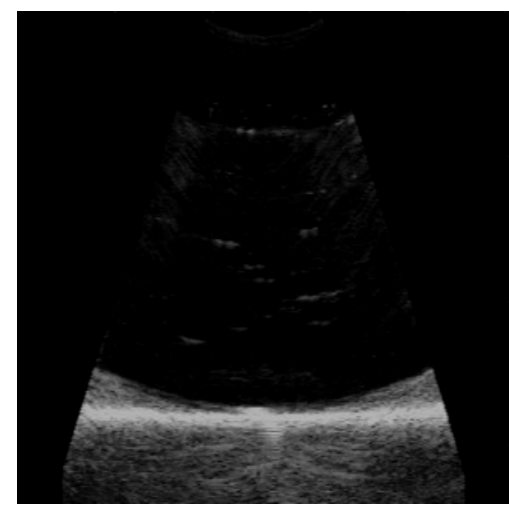

(a)

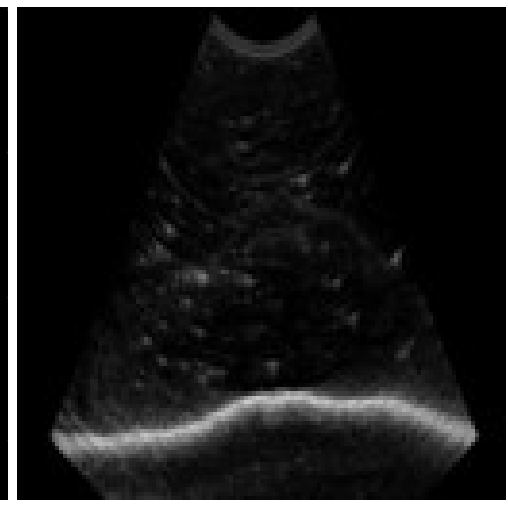

(b)

Figs. 7.Influence of riverbed on the imaging spots during particles begin to go on incipient motion, (a)horizontal and smooth riverbed filled with plastic sand particles at the flow velocity of $4.2 \mathrm{~cm} / \mathrm{s}$, (b)steep and rough riverbed filled with the same sediment particles at the flow velocity of $4.2 \mathrm{~cm} / \mathrm{s}$

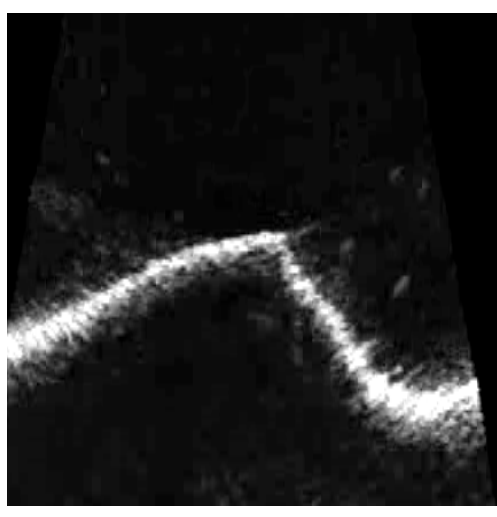

(a)

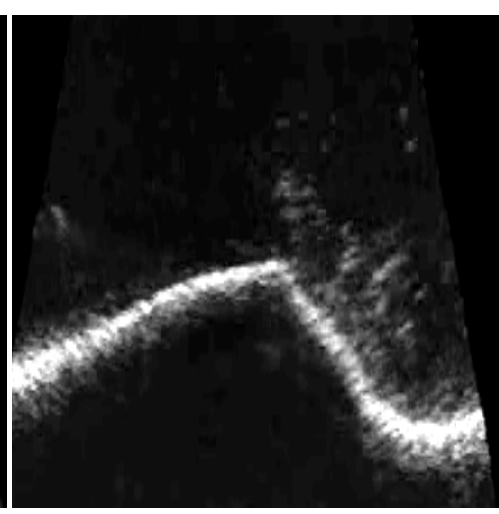

(b)

Figs. 8.Influence of flow conditions on the imaging spotsduring lots of particles go on incipient motion, steep slope consisted of plastic sands at the flow velocity of $5.3 \mathrm{~cm} / \mathrm{s}(\mathrm{a})$ and of $8.6 \mathrm{~cm} / \mathrm{s}(\mathrm{b})$

From Figs. 7-8, we can see that different river beds and flow conditions can affect the movement states of sediment particles near the riverbed. Whenwater flow velocity ishigher,the horizontal and smooth riverbed is the easiest to make sediment 
particles to go on incipient motion, especially when topography riverbed exist a steep slope. However, these vary conditions are difficult to be controlled at the same time and under the same situation in practice. And their influences on the SIV are complex and interconnected each other. They are difficult to be sorted out and meaningless for the actual engineering application. So, using B-scan ultrasound imaging method has a big advantage in actual model experiments, but this method can direct reflect the sediment movement and monitor the process of sand incipient motion. By statistical analysis, the SIV is easy to be obtained by analysing the number/area of imaging spots from underwater sediment particles.

In order to show the effect of water flow on the results, we have reorganized a part of original statistical data. The original data are from the statisticalprocess of analysing the imaging spot number of plastic sands, i.e., the data of row $N$-ps- 0.1 in Table 1. One value in the table is the mean value of 50 or more original statistical values. We went on statistical analysis for the relative error of each value in the row $N$-ps-0.1 of Table 1. The results are shown in Fig. 9. The standard deviation $\delta$ in Fig. 9 is defined as formula (5). Thei, $j, m$ and $n_{j}$ is the same as formula (1) and the $\delta_{i}$ is the standard deviation of $i$-th row. 
From Fig. 9, we can see that statistical mean value become more stable when the water flow velocity is more close to the SIV of $0.1 \mathrm{~mm}$ plastic sands, i.e., the above measured SIV $3.7 \mathrm{~cm} / \mathrm{s}$ from the $N$-ps-0.1. It means the measured SIV 3.7 $\mathrm{cm} /$ sfor plastic sands whose particle size is $0.1 \mathrm{~mm}$ is suitable and also the determining of the SIV at the sudden change point of the curve is reasonable.The measurement method of the SIV can be acceptable.

Another, there is a peak point K $(7.81,20.18)$ in the curve of natural sands (i.e., the point K of curve Rn-ns-0.3 in Fig. 5b.This phenomenon is the incipient motion of non-uniform sediment in the river model experiments[32, 33]. At this point, some heavier and bigger sands are starting to move away, which are remained particles after mostly lightersediment particles have been moved away from the riverbed. When the granulometric distribution of sediment particles is controlled well, this method can be also used to analysis and research the incipient motion of non-uniform sediment in future.

\subsection{Verification of the measured SIV}

In order to further validate whether the above measured SIVsof plastic/natural sands (e.g., $3.7 \mathrm{~cm} / \mathrm{s}$ of $N$-ps-0.1,4.2 cm/s of $N$-ns-0.1, $4.9 \mathrm{~cm} / \mathrm{s}$ of $N$-ps-0.2, and 5.5 cm/s of $N$-ns-0.3 in Figs. 5) areright or not,one group ofriverbed'sboundary lines are obtained by image processing method[34]. Taking plastic sandswhose particle size is $0.1 \mathrm{~mm}$ as example, the change of riverbed under different flow velocities are shown in Figs. 10. If flow velocity is up to the SIV of $0.1 \mathrm{~mm}$ plastic sands, the particles near the riverbed can start to move from the river bed, and then the boundary lines of the bed begin to change also. Therefore, the changed boundary lines can be used to validate the above measured SIV or not. Among Figs. 10, Fig. 10a shows the original boundary line of riverbed which is consisted of plastic sands under the flow velocity of $2.3 \mathrm{~cm} / \mathrm{s}$; Fig. $10 \mathrm{~b}$ shows the boundary line of the riverbed when the flow velocity is up to $3.3 \mathrm{~cm} / \mathrm{s}$, which is obtained after 30 minutes;Fig. 10c shows the boundary line of the riverbed when the flow velocity is up to $4.0 \mathrm{~cm} / \mathrm{s}$, which is obtained after 30 minutes also at the same condition. 


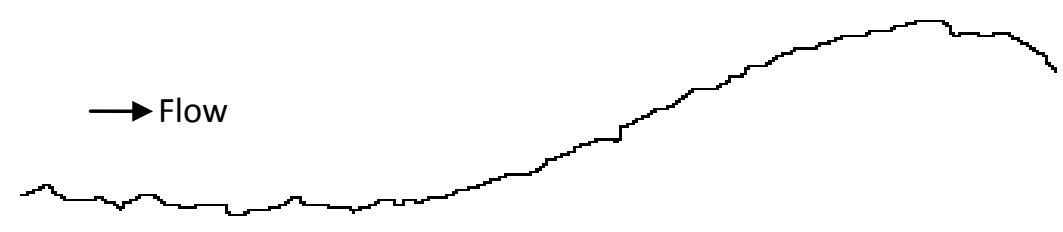

(a)

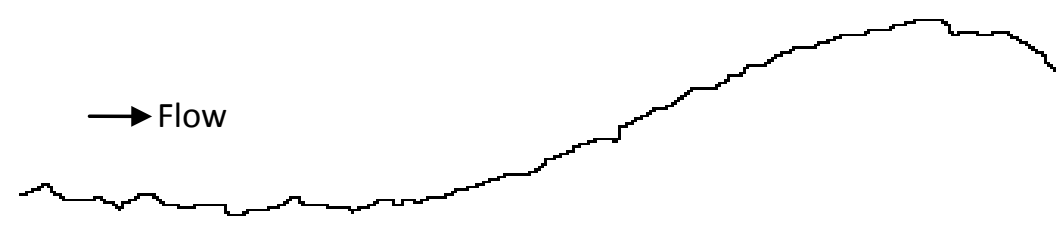

(b)

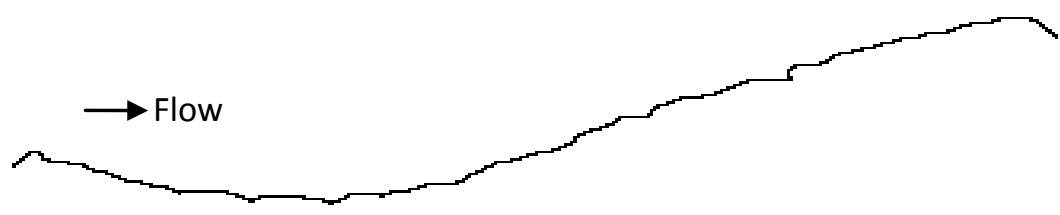

(c)

Figs. 10.Comparison of riverbed boundary linesunder different flow velocities after 30 minute, (a) is under flow velocity $2.3 \mathrm{~cm} / \mathrm{s}$, (b) is under flow velocity $3.3 \mathrm{~cm} / \mathrm{sand}$ (c) is under flow velocity $4.0 \mathrm{~cm} / \mathrm{s}$

From Figs. 10a-10b, the boundary lines of riverbed are nearly the same as before. They remain unchanged when flow velocity is in the range of 2.3 to $3.3 \mathrm{~cm} / \mathrm{s}$. It means that the plastic sand particles almost remain motionless. When flow velocity is up to $4.0 \mathrm{~cm} / \mathrm{s}$ and remains unchanged for 30 minutes, the boundary line of riverbed changes obviously shown inFig. 10c compared with Figs. 10a-10b. The peak of riverbed boundary line becomes flat, and moves to the right side. When flow velocity decreases to $3.3 \mathrm{~cm} / \mathrm{s}$, the boundary line is still changing after 30 minutes. Therefore, the measured SIV (e.g., $3.7 \mathrm{~cm} / \mathrm{s}$ ) of plastic sands (particle size $0.1 \mathrm{~mm}$ ) is reasonable, which is within the range of $3.3 \mathrm{~cm} / \mathrm{sto} 4.0 \mathrm{~cm} / \mathrm{s}$. This measured result is acceptable in practice.

\section{Conclusion}

This paper takes four kinds of sediment particles as examples to show a novel method of measuring the SIV by using B-scan ultrasound imaging device, and the first applicationin the analysis of the whole sediment incipient motionprocess inthe river model experiments. Several groups of comparative experiments are alsotaken to show the measurement process of the SIV. Through experiments and analysis,the curves of statistical imaging spots shown in Figs. 5-6 have clearly disclosed the 
process of sediment incipient motion duringour water channel tests. The suddenly changed positions of the curves can correspond to the sediment incipient motion. And the flow velocities of these positions exactlycorrespond to the SIVs, e.g., the SIV of $0.1 \mathrm{~mm}$ plastic sands is between A $(3.2 \mathrm{~cm} / \mathrm{s})$ and $\mathrm{E}(4.1 \mathrm{~cm} / \mathrm{s})$, the SIV of $0.1 \mathrm{~mm}$ natural sands is between $B(3.8 \mathrm{~cm} / \mathrm{s})$ and $F(4.5 \mathrm{~cm} / \mathrm{s})$, the SIV of $0.2 \mathrm{~mm}$ plastic sands is between C $(4.4 \mathrm{~cm} / \mathrm{s})$ and $\mathrm{G}(5.3 \mathrm{~cm} / \mathrm{s})$ and the SIV of $0.3 \mathrm{~mm}$ natural sands isbetween D $(5.1 \mathrm{~cm} / \mathrm{s})$ and $\mathrm{H}(5.8 \mathrm{~cm} / \mathrm{s})$. We take their mid-values (i.e., $3.7 \mathrm{~cm} / \mathrm{s}, 4.2$ $\mathrm{cm} / \mathrm{s}, 4.9 \mathrm{~cm} / \mathrm{s}, 5.5 \mathrm{~cm} / \mathrm{s}$ ) as their SIVs, respectively. This method is especially suitable for the measurement of SIV in sediment-laden flow or muddy water when flow transparency is too poor to see the moving particles in the water clearly.

Another, this B-scan ultrasound imaging device has the advantages of high resolution and strong penetrability. It can directly observe sediment particles as small as particle size $0.1 \mathrm{~mm}$ under the water. If the granulometric distribution of sediment particles is controlled well, this method is suitable for the analysis and research of non-uniform sediment incipient motion. This device and B-scan ultrasound imaging measurement method can be used to monitor the sediment movementandthe bottom riverbed development in sediment-laden flow or under muddy water. It has a high value of practical application and is playing an important role during the river model experiments for sediment engineering. This is a novel approach for the research of sediment movement and fluvial process, and will bring convenience and progress for many researches.

\section{Acknowledgments}

This paper was supported by the Strategic Research Program of the Chinese Academy of Sciences (Grant No.XDB10030200), the National Science Foundation of China (Grant No. 41402278), and also the Fundamental Research Funds for the Central Universities (2014212020205).Another, some experimental materials come from Yangtze River Scientific Research Institute and Wuhan University. We thank their kind help very much. Finally, we are grateful for the many helpful and constructive comments from my supervisor and many anonymous reviewers. 


\section{References}

[1] S.J. Childs, Incipient motion criteria for a rigid sediment grain on a rigid surface, Journal Of Engineering Mechanics-Asce, 127 (2001) 524-529.

[2] T.S. Yang, Q.S. Li, Z.H. Wan, Incipient motion of fine sediment particles in flow of large depth, Hydraulics Of Rivers Water Works And Machinery, Vol Ii, Theme D, Proceedings: 21st Century: The New Era for Hydraulic Research And Its Applications, 2001, pp. 152-160.

[3] S. Joshi, G.P. Duffy, C. Brown, Settling Velocity And Grain Shape Of Maerl Biogenic Gravel, Journal Of Sedimentary Research, 84 (2014) 718-727.

[4] J.P. Prancevic, M.P. Lamb, B.M. Fuller, Incipient sediment motion across the river to debris-flow transition, Geology, 42 (2014) 191-194.

[5] J. Yang, X. Hou, H. Li, S. Cao, X. Liu, The Nonlinear Cusp-catastrophe Model of Incipient Motion for Non-uniform Sediment, Proceedings Of the 35th Iahr World Congress, Vols I And Ii, (2013) 3792-3797.

[6] J.E. Ball, A. Keshavarzy, Incipient Sediment Motion on Non-Horizontal Slopes - Discussion, Journal Of Hydraulic Research, 33 (1995) 723-724.

[7] B. Bohling, Measurements of threshold values for incipient motion of sediment particles with two different erosion devices, Journal Of Marine Systems, 75 (2009) 330-335.

[8] J.C. Curran, K.A. Waters, K.M. Cannatelli, Real time measurements of sediment transport and bed morphology during channel altering flow and sediment transport events, Geomorphology, 244 (2015) 169-179.

[9] Y. Lu, Y.-j. Lu, Y.-m. Chiew, Incipient motion of cohesionless sediments on riverbanks with ground water injection, International Journal Of Sediment Research, 27 (2012) 111-119.

[10] B.M. Crookston, B.P.M. Tullis, Incipient motion of gravel in a bottomless arch culvert, International Journal Of Sediment Research, 26 (2011) 15-26.

[11] H. Zhang, A unified formula for incipient velocity of sediment, Journal of Hydraulic Engineering, 43 (2012) 1387-1396.

[12] H.W. Tang, H. Wang, D.F. Liang, S.Q. Lv, J. Yan, Incipient motion of sediment in the presence of emergent rigid vegetation, Journal Of Hydro-Environment Research, 7 (2013) 202-208.

[13] Y. Bai, X. Wang, Y. Cao, Incipient motion of non-uniform coarse grain of bedload considering the impact of two-way exposure, Science China-Technological Sciences, 56 (2013) 1896-1905.

[14] N.-m.M. Hossein, K. Hossein, M.-S. Rasoul, Laboratory analysis of incipient motion velocity for non-uniform non-cohesive sediments movement in rectangular flumes, Arabian Journal Of Geosciences, 9 (2016).

[15] Y.C. Bai, C.O. Ng, H.T. Shen, S.Y. Wang, Rheological properties and incipient motion of cohesive sediment in the Haihe Estuary of China, China Ocean Engineering, 16 (2002) 483-498.

[16] B. Kumar, G. Srinivasulu, A.R. Rao, Incipient motion design of sand bed channels affected by bed suction, Computers And Electronics In Agriculture, 74 (2010) 321-328.

[17] L. Dong-Hyeok, C. Nahm-Gyoo, Assessment of surface profile data acquired by a stylus profilometer, Measurement Science and Technology, 23 (2012) 105601.

[18] C. Cui, X. Xu, H. Huang, J. Hu, R. Ye, L. Zhou, C. Huang, Extraction of the grains topography 
from grinding wheels, Measurement, 46 (2013) 484-490.

[19] P. Pawlus, Digitisation of surface topography measurement results, Measurement, 40 (2007) 672-686.

[20] C. Di Cristo, M. Greco, M. Iervolino, A. Leopardi, A. Vacca, Two-Dimensional Two-Phase Depth-Integrated Model for Transients over Mobile Bed, Journal Of Hydraulic Engineering, 142 (2016).

[21] M. Crapper, T. Bruce, C. Gouble, Flow field visualization of sediment-laden flow using ultrasonic imaging, Dynamics Of Atmospheres And Oceans, 31 (2000) 233-245.

[22] X.-j. Zou, Z.-m. Ma, X.-h. Zhao, X.-y. Hu, W.-l. Tao, B-scan ultrasound imaging measurement of suspended sediment concentration and its vertical distribution, Measurement Science And Technology, 25 (2014).

[23] X.-j. Zou, Z.-m. Ma, W.-b. Hu, J.-c. Wang, H. Song, X.-y. Hu, W.-l. Tao, B-mode ultrasound imaging measurement and 3D reconstruction of submerged topography in sediment-laden flow, Measurement, 72 (2015) 20-31.

[24] K.-C. Fan, J. Torng, W. Jywe, R.-C. Chou, J.-K. Ye, 3-D measurement and evaluation of surface texture produced by scraping process, Measurement, 45 (2012) 384-392.

[25] X. Zou, H. Song, C. Wang, Z. Ma, Relationships between B-mode ultrasound imaging signals and suspended sediment concentrations, Measurement, 92 (2016) 34-41.

[26] L.-P. Liang, K.-J. Xu, X.-F. Wang, Z. Zhang, S.-L. Yang, R. Zhang, Statistical modeling and signal reconstruction processing method of EMF for slurry flow measurement, Measurement, 54 (2014) 1-13. [27] N.A. Marsh, A.W. Western, R.B. Grayson, Comparison of methods for predicting incipient motion for sand beds, Journal Of Hydraulic Engineering-Asce, 130 (2004) 616-621.

[28] D. Pal, K. Ghoshal, Grain-size distribution in open channel flow by mixing length approach, Environmetrics, 26 (2015) 107-119.

[29] A. Haddadchi, N. Movahedi, E. Vahidi, M.H. Omid, A.A. Dehghani, Evaluation of suspended load transport rate using transport formulas and artificial neural network models (Case study: Chelchay Catchment), Journal Of Hydrodynamics, 25 (2013) 459-470.

[30] C.T. Yang, R. Marsooli, M.T. Aalami, Evaluation of total load sediment transport formulas using ANN, International Journal Of Sediment Research, 24 (2009) 274-286.

[31] A. Motamedi, H. Afzalimehr, V.P. Singh, Evaluation of a Novel Approach to Determine the Critical Shields Stress, Journal Of Hydrologic Engineering, 15 (2010) 892-900.

[32] Y. Fengguang, C. Shuyou, L. Xingnian, Y. Kejun, Study on Incipient Velocity of Individual Fradctions of Non-uniform Sediment, Journal of Sichuan University. Engineering Science Edition, 40 (2008) 51-57.

[33] R.S. Pugh, I.N. McCave, Particle Size Measurement Of Diatoms with Inference Of Their Properties: Comparison Of Three Techniques, Journal Of Sedimentary Research, 81 (2011) 600-610.

[34] P. Demircioglu, Estimation of surface topography for dental implants using advanced metrological technology and digital image processing techniques, Measurement, 48 (2014) 43-53. 


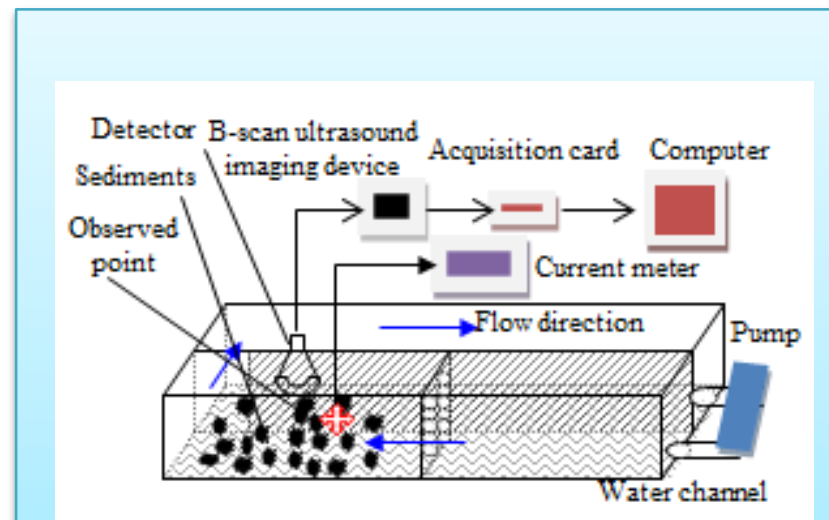

Sediment incipient motion experiments

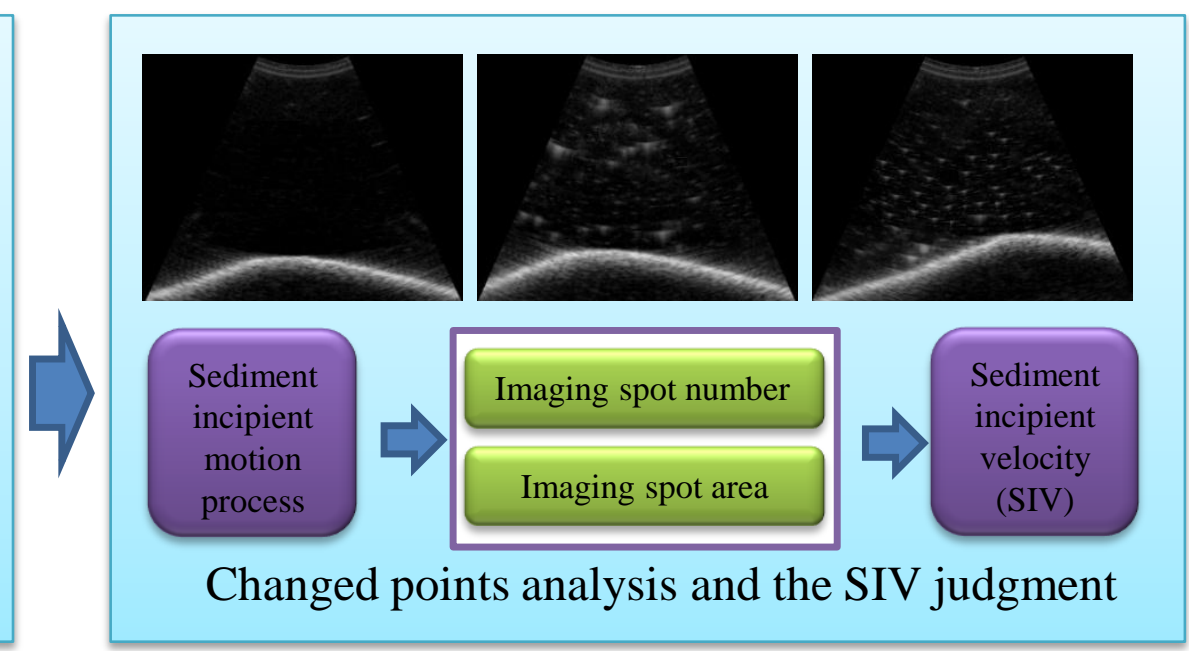

